Prawo Kanoniczne

$56(2013) \mathrm{nr} 4$

ANTONIO INTERGUGLIELMI

Studium Theologicum Galilaeae, Israel

\title{
IL RUOLO DEGLI ORGANI DI PARTECIPAZIONE PREVISTI NEL CODICE DI DIRITTO CANONICO: GLI ATTI DI AMMINISTRAZIONE STRAORDINARIA E L'ALIENAZIONE DI BENI DEGLI ENTI ECCLESIASTICI
}

Sommario: Premessa. - 1. Atti di alienazione e atti di straordinaria amministrazione. 2. Le indicazioni del Codice di Diritto Canonico del 1983: i beni ecclesiastici, il concetto di "patrimonio stabile" e gli atti di amministrazione straordinaria e di alienazione. - 2.1. Atti di alienazione. - 2.2. Atti di straordinaria amministrazione. - 3. Il ruolo degli Organismi di partecipazione verso gli atti di amministrazione straordinari dell'Ente ecclesiastico: Consiglio diocesano per gli affari economici (CDAE) e Consiglio Parrocchiale affari economici (CPAE). - 4. Alcune situazioni particolari di atti peggiorativi del patrimonio. -5. Gli atti di straordinaria amministrazione della Parrocchia: la licenza canonica. - 6. Ruolo del Consiglio parrocchiale per gli affari economici (CPAE). - Conclusioni.

\section{Premessa}

I beni temporali nella vita della Chiesa hanno un valore essenzialmente strumentale: l'amministrazione è, infatti, l'insieme delle attività che hanno come scopo l'organizzazione del patrimonio riguardo a un fine.

La Chiesa possiede e amministra i beni temporali solo perché mezzi per raggiungere gli obiettivi che le sono propri: il sostegno delle opere di apostolato e di carità (cfr. PO 17 e can. 1254 § 2), nonché la cura del culto e dei luoghi sacri.

Il raggiungimento di queste finalità suscita e giustifica una particolare attenzione da parte della Chiesa per la corretta amministrazione dei suoi beni. 
Tuttavia spesso la responsabilità del patrimonio ecclesiastico potrebbe essere completamente affidata a persone prive di una particolare preparazione nella gestione, quali spesso sono i chierici: si pensi ad un Parroco che, privo di qualsiasi competenza economica, si ritrovi a gestire insieme alla Parrocchia, una scuola o addirittura una clinica che dipenda da essa, o anche semplicemente una serie di immobili di proprietà della stessa.

E' anche per questa ragione che nel Codice di diritto canonico del 1983 (da ora anche CIC/83), vengono introdotti una serie di organismi, i Consigli economici, con la funzione non solo di controllare l'aspetto economico con cui viene svolta la gestione, ma anche con l'intento di assicurare una collaborazione e un supporto nell'amministrazione dei beni ecclesiastici ${ }^{1}$.

$\mathrm{Ci}$ si è resi infatti conto che la sempre maggiore complessità del mondo economico-amministrativo esige oramai la collaborazione di persone qualificate ed esperte che, con il loro prezioso aiuto e sapiente consiglio, favoriscano una buona e corretta amministrazione dei beni e una più razionale e moderna gestione degli stessi.

Gli atti di amministrazione dei beni sono espressione del potere di governo esecutivo, attribuito all'autorità che ha potestà esecutiva rispetto a un ente ecclesiastico. Potere quindi collegato alla facoltà di porre atti amministrativi sui beni ${ }^{2}$.

${ }^{1}$ Questi nuovi organismi di partecipazione dei fedeli al governo, anche economico, dalla comunità ecclesiale, sono espressione dell'attuazione dei principi del Concilio Vaticano II: in particolare il nr. 30 del decreto Christus Dominus, dove si afferma che „Nell'esercizio della loro missione i parroci con i loro cooperatori devono svolgere il compito di insegnare e governare in modo che i fedeli e le comunità parrocchiali si sentano realmente membri non solo della diocesi, ma anche della Chiesa Universale". Decreto sull'Ufficio Pastorale dei Vescovi nella Chiesa, Christus Dominus, 28 ottobre 1965, AAS 58(1966), pp. 673-701.

2 Cfr. V. De Paolis, I beni temporali della Chiesa, ed. EDB, Bologna, 2005, p. 77 , dove precisa che „Il can. 1254 rivendica il diritto ai beni attraverso quattro verbi: acquirere, retinere, administrare et alienare. Non c'è dubbio che ognuno di questi atti esprime un potere di governo sulle cose". Sempre dello stesso autore, De bonis Ecclesiae temporalibus. Adnotationes in Codicem: Liber V, Roma 1986. 
Il criterio adottato dal codice, in base al can. 116, di imporre il controllo dell'autorità superiore per gli atti di straordinaria amministrazione a tutela della stabilità del patrimonio, viene quindi supportato e rafforzato dall'esistenza di questi Consigli, composti anche da laici.

Ma per meglio trattare la questione della responsabilità canonica nell'amministrazione, occorre approfondire la distinzione che il CIC/83 accoglie, distinguendo il concetto dell'atto di alienazione (cann. $1291 \mathrm{e}$ 1295) da quello degli atti di straordinaria amministrazione (cann. 1277 e 1281).

\section{Atti di alienazione e atti di straordinaria amministrazione}

Nella legislazione canonica dedicata al diritto patrimoniale, ai fini della salvaguardia dei beni si è sempre ritenuto necessario operare la distinzione tra patrimonio stabile e patrimonio fluttuante, intendendo con la prima espressione quei beni che garantiscono alla persona giuridica pubblica il raggiungimento del proprio fine. Questi beni sono sottoposti alla legislazione sulle alienazioni.

Pertanto, anche nel nuovo Codice di diritto canonico, per gli atti di amministrazione che riguardano $i$ beni temporali, si ritrova la grande distinzione tra atti di alienazione e atti di straordinaria amministrazione. Tuttavia bisogna rilevare che in dottrina è aperto il dibattito i primi possano farsi rientrare nel concetto di atti di straordinaria amministrazione.

L'incertezza nasce da una certa contraddizione terminologica delle norme del CIC: sebbene il nuovo CIC contenga una normativa specifica per gli atti di amministrazione e per quelli di alienazione, in altra parte definisce però gli atti di alienazione come atti di amministrazione straordinaria ${ }^{3}$.

Malgrado queste incertezze terminologiche è indubbio che il CIC/83 compie notevoli progressi nella chiarificazione della distinzione tra atti di amministrazione straordinaria e atti di alienazione, poiché il codice piano-Benedettino disciplinava solo gli atti di alienazione e non anche quelli di amministrazione straordinaria.

${ }^{3}$ Nei lavori preparatori del codice si disse espressamente: ,quia alienatio non est actus administrationis", Communicationes 12(1980), p. 396. 
Il CIC/83 identifica dunque gli atti di amministrazione straordinaria come quegli atti che non possono essere compiuti ad validitatem sen-

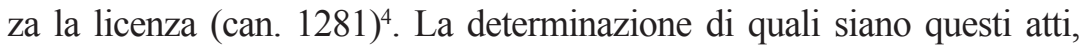
è rimessa al Vescovo diocesano, agli Statuti o alla Conferenza episcopale.

Tutti gli atti di una certa importanza che non sono atti di alienazione devono quindi sottostare a un controllo specifico. Il codice prescrive i modi d'individuazione di questi atti: la Conferenza episcopale per il Vescovo diocesano (can. 1277) $)^{5}$; il Vescovo diocesano per le persone a lui soggette (can. $1281 \S 2$ ), in linea con gli statuti delle stesse ${ }^{6}$. Il can. 1295 stabilisce, infatti, che negli statuti delle persone giuridiche deve essere inserita la normativa sulle alienazioni (cann. 1291-1294). Queste norme non valgono per qualunque atto di straordinaria amministrazione, ma solo per quelli che si riferiscono a beni che fanno parte del patrimonio stabile e che hanno per oggetto un diritto reale.

\section{Le indicazioni del Codice di Diritto Canonico del 1983:} i beni ecclesiastici, il concetto di "patrimonio stabile" e gli atti di amministrazione straordinaria e di alienazione

Il Codice del 1983 stabilisce che solo le persone ecclesiastiche pubbliche hanno la capacità di essere titolari di beni ecclesiastici: i beni delle persone giuridiche private non sono beni ecclesiastici, per cui non sono sottoposti alle regole del diritto universale del libro $\mathrm{V}$ del CIC, né a quelle riguardanti il concetto di patrimonio stabile. Ovviamente il dovere di vigilanza dell'Ordinario è molto penetrante per le persone giuridiche pubbliche che agiscono in nome della Chiesa rispetto agli enti ecclesiali privati.

Il CIC/83, al can. $1276 \S 1$, attribuisce all'Ordinario la tutela dell'amministrazione dei beni appartenenti alle persone giuridiche pubbliche a lui soggette. Sempre 1'Ordinario ha il diritto-dovere di vi-

${ }^{4}$ Can. $1281 \S 1$ :,fines et modum ordinariae administrationis excedunt".

5 Cfr. F. G. Morrisey, Ordinary and extraordinary administration:canon 1277, The Jurist 48(1988), pp. 709-726.

${ }^{6}$ Per gli istituti religiosi sono gli statuti degli stessi a determinarli (can. $638 \S 1$ ). cfr. J. Beyer, Le droit de la vie consacréè. Instituts et Sociétés, Paris 1988. 
gilare sugli altri enti privati, nei limiti previsti dalle norme del Codice stesso (cann. $392 \S 2,305,325 \S 1$ ). Il riferimento non si limita al Vescovo diocesano, ma include gli altri Ordinari della Chiesa particolare: il Vicario generale e il Vicario episcopale. Questa normativa inoltre si applica anche agli altri Ordinari di cui al can. $134 \S$ 1, tra cui i Superiori maggiori degli Istituti religiosi e le Società di vita apostolica clericali di diritto pontificio.

Rispetto a questi ultimi, la vigilanza dell'autorità superiore consiste soprattutto nel controllare il rispetto delle disposizioni dello Statuto della persona giuridica privata, in particolare per quanto concerne l'utilizzo dei beni per i fini stabiliti (cann. 305 e $325 \S 1$ ).

Il codice per la disciplina sull'alienazione, al can. 1291, parla di patrimonio stabile ${ }^{7}$ : si tratta principalmente di beni immobili, ma anche denaro depositato o investito in azioni societarie o titoli di credito. Non ci sono regole per la fissazione di un patrimonio stabile per cui la novità del codice sta proprio nel disporre la necessità di un atto di assegnazione.

La presunzione è perciò che un bene non faccia parte del patrimonio stabile sino a quando non si provi il contrario. Tuttavia, vi sono dei beni che, per la loro natura, sono da considerare come facenti parte del patrimonio stabile: sono quelli senza dei quali la persona giuridica non avrebbe i mezzi per il perseguimento dei propri fini istituzionali ${ }^{8}$.

7 L'espressione patrimonio stabile non era presente nel CIC del 1917 se non al can. $1530 \S 1$, in cui c'era l'espressione res ecclesiasticae immobiles aut mobiles quae servando servari possunt, riferendosi in particolare ai beni immobili. La nuova espressione scelta, beni legittimamente assegnati al patrimonio stabile, accoglie tra l'altro una mutata realtà economica, dove la distinzione tra beni immobili e mobili non è sempre facilmente determinata.

${ }^{8}$ In dottrina già il Wernz rivendica il diritto della Chiesa di alienare i beni, ma allo stesso tempo lo circoscrive entro limiti rigidi: „Nam ex iure divino nullus administrator bonorum alienorum sine iusta caus valide alienare potest", WERNZ, Ius canonicum, n. 758. Nel CIC/83, al canone 1285, si afferma inoltre esplicitamente che anche i beni mobili possono far parte del patrimonio stabile: ,gli amministratori possono fare donazioni a fini di pietà e di carità cristiana dei beni mobili non appartenenti al patrimonio stabile". 
In dottrina, Arturo Tabera, ancor prima del codice del 1983, definiva patrimonio stabile come il complesso di "beni che costituiscono quasi la base del sostentamento della persona", e vi includeva anche il capitale che produce un reddito che permette all'Ente ecclesiastico di svolgere la propria attività: in questo caso questo denaro dovrà considerarsi come dotato di una "certa immutabilità"9.

Un bene è legittimamente assegnato a una persona giuridica quando alla stessa sia stato attribuito, con disposizione normativa, un provvedimento dell'autorità ecclesiastica competente, con delibera degli amministratori o per altro titolo (es. tavole di fondazione o volontà dei donanti).

L'alienazione è così distinta dall'amministrazione anche per le conseguenze nel caso di invalidità dell'atto posto in essere privo delle solennità previste dal CIC: il can. 1256, riprendendo sostanzialmente il contenuto del can. 1534 del Codice di diritto canonico del 1917 (da ora anche CIC/17), prevede un'azione per i beni invalidamente alienati, mentre non si trova alcuna norma specifica per gli atti di amministrazione straordinaria posti invalidamente.

L'alienazione è la disposizione del bene fino a perderne in tutto o in parte il dominio (can. $1254 \S 1$ ); si distingue quindi dall'amministrazione.

\subsection{Atti di alienazione}

L'alienazione è la cessione della proprietà da parte di una persona giuridica canonica pubblica, perché solo a questa appartengono i beni ecclesiastici: a questo riguardo De Paolis afferma: "Con l'alienazione i beni cessano di essere ecclesiastici e ritornano al campo profano, e non sono più a servizio della Chiesa. Da questo punto di vista la legislazione in proposito è nata in funzione proprio di proibire l'alienazione dei beni ecclesiastici"10.

L'alienazione è trattata in maniera distinta dal codice perché incide sul patrimonio diminuendolo di un diritto fondamentale: quello di

\footnotetext{
9 A. TABERA, Il diritto dei religiosi, Roma 1961, p. 191.

${ }^{10} \mathrm{~V}$. De Paolis, I beni temporali, cit., p. 184.
} 
proprietà. Si tratta sempre di un atto che oltrepassa fines modumque l'ordinaria amministrazione.

L'alienazione si può realizzare in senso stretto o in senso ampio ${ }^{11}$. In senso stretto quando si trasferisce il dominio diretto dalla persona giuridica canonica ad altro titolare, che può essere anche un'altra persona giuridica canonica (cann. 1291-1294); in senso ampio quando pur non trasferendo il dominio diretto sulla cosa, si concede un diritto reale sulla stessa, in modo che il dominio diretto ne è diminuito o comunque condizionato (can. 1295).

Questa distinzione nasce dal contenuto dei canoni del CIC/17 che parlavano di alienazione in senso proprio (can. 1530), includendovi il solo trasferimento della proprietà, e alienazione in senso improprio (can. 1533), con cui si consideravano gli atti traslativi di un diritto reale sulla proprietà ${ }^{12}$.

Altri beni che ricadono sotto la disciplina delle alienazioni sono gli ex-voto donati alla Chiesa, le cose preziose per ragioni storiche o artistiche (can. $1292 \S 2$ ), le sacre reliquie.

Per la validità dell'atto il Codice al can. 1293 esige una giusta cau$\mathrm{sa}^{13}$, che deve consistere in un'urgente necessità, evidente utilità, motivo di pietà o di carità o qualche altra grave ragione pastorale.

Ai concetti classici di utilitas, necessitas, pietas si aggiungono dunque due concetti più attuali: la carità e una grave ragione pastorale.

L'autorità che è tenuta a rilasciare l'autorizzazione (licenza) deve valutare l'opportunità dell'alienazione affinché quest'ultima sia proporzionata con la causa.

In questo senso la justa causa, non essendo un elemento strutturale del contratto, si pone come un presupposto di fatto del negozio: l'alienazione, infatti, non sarà ritenuta invalida per l'inesistenza del presup-

${ }^{11}$ Altra distinzione che si ritrova in dottrina è tra alienazione in senso proprio e alienazione in senso improprio.

${ }^{12}$ Questa terminologia si rivela poco chiara: riteniamo che sia per questa ragione che i riformatori del codice di diritto canonico abbiano preferito omettere la qualifica „,proprie dicta”, del can. 1533 del CIC/17.

${ }^{13}$ Quando manca una giusta causa l'atto deve considerarsi nullo per mancanza di un elemento essenziale. 
posto rispetto all'atto (o al contratto) mediante il quale l'alienazione è attuata, ma piuttosto perché verrà meno la possibilità di concedere la licenza che il legislatore richiede. Questo quando il valore dell'alienazione eccede la quantità minima legalmente determinata.

L'analisi della giusta causa è così l'elemento principale che consente di individuare l'opportunità del rilascio della licenza ad alienare da parte del Vescovo diocesano. Non solo. La giusta causa sarà anche oggetto di analisi da parte del Consiglio per gli affari economici e del Collegio dei consultori, affinché sia concesso al Vescovo diocesano il consenso al rilascio della licenza.

Altro elemento per la validità della donazione è stabilito dal $\S 3$ del can. 1293: nel caso in cui il bene sia divisibile, devono essere indicate le parti del bene stesso che sono state oggetto di alienazione in precedenza: la licenza sarà nulla nel caso in cui non siano state date al Vescovo queste informazioni. E' chiaro che la divisibilità del bene dipende sempre dalle regole di diritto civile cui bisogna fare riferimento. Rimangono, comunque, su questo aspetto punti ancora da chiarire: sarà necessario fare riferimento a qualsiasi alienazione fatta, anche minima? E ancora. Qual è il tempo della memoria storica che deve essere considerato?

Per queste ragioni, il Vescovo diocesano è opportuno che faccia sempre un'integrazione alla normativa particolare, compito che al Vescovo è dato in attuazione al dovere più generale stabilito nel can. 1276 $\S 2$ : “emanare speciali istruzioni finalizzate a ordinare l'intero affare dell'amministrazione dei beni" 14 .

Infine il can. $1292 \S 2$ richiede che, se il valore dei beni superasse una determinata somma, la stima della cosa da alienare deve essere fatta con una perizia. Non bisogna infatti qui dimenticare che si tratta della valutazione periziale del bene e non del prezzo. Quest'ultimo potrebbe dunque variare rispetto alla valutazione fatta dai periti. Al riguardo, il valore periziale obbliga l'alienante alla richiesta della licenza all'Autorità superiore anche qualora il bene sia in seguito venduto

${ }^{14}$ Così, C. Redaelli, La responsabilità del Vescovo diocesano nei confronti dei beni ecclesiastici, Quaderni di Diritto Ecclesiale 4(1991), p. 327. 
a un prezzo inferiore. Al contrario, se una perizia stabilisce il valore del bene sotto il limite minimo con cui si richiede l'atto autorizzativo, l'alienante non deve chiedere la licenza, anche se il bene sarà poi venduto $\mathrm{a}$ un prezzo più alto del valore minimo ${ }^{15}$.

\subsection{Atti di straordinaria amministrazione}

La distinzione tra amministrazione ordinaria e straordinaria non è mai stata semplice: alcuni autori, rispetto ai canoni del CIC/17, risolvevano la questione in modo semplicistico affermando che serve, per gli atti straordinari, la licenza del Superiore (con riferimento al can. $532 \S 2 \mathrm{CIC} / 17)$.

In realtà l'amministrazione non è da considerarsi straordinaria perché è richiesta la licenza, ma perché si tratta di un atto peggiorativo del patrimonio della persona giuridica canonica che intende porlo in essere. La licenza è dunque una conseguenza della natura dell'atto, e non un criterio di distinzione degli atti.

Anche il CIC/83 conserva però il criterio del precedente Codice "piano benedettino": 1'atto non è definito in assoluto ma riguardo all'amministrazione ordinaria (can. $1281 \S 1$ ).

Il $\S 2$ dello stesso canone fornisce, inoltre, una precisazione fondamentale: saranno gli statuti o il Vescovo diocesano, a stabilire gli atti eccedenti l'amministrazione ordinaria.

Occorre rifarsi a questo paragrafo dunque per individuare in pratica gli atti soggetti a licenza. Allo stesso tempo il can. $638 \S 1$ rimanda al "diritto proprio" la determinazione degli atti che eccedono il limite e le possibilità dell'amministrazione ordinaria, pur nell'ambito del diritto universale.

L'Ordinario interviene così non solo per quegli Enti il cui statuto non fornisce alcuna indicazione ma anche per tutte le persone giuridiche sotto la sua giurisdizione, in primis le parrocchie (can. $1281 \S 2$ ).

Di fatto il Codice combina criteri teorici e criteri pratici con riferimento alla situazione particolare delle diverse persone giuridiche.

\footnotetext{
${ }^{15}$ Cfr. J. L. SAntos Diez, Administration exstraórdinaria de los bienes ecclesiásticos, in: XIX Semana Española de Derecho Canónico, Salamanca 1985, p. 49.
} 
Per la Diocesi, invece, sarà la Conferenza Episcopale a stabilire quali atti siano da considerare di straordinaria amministrazione.

Abbiamo, dunque, con il nuovo CIC una più chiara distinzione tra i due atti: gli atti di alienazione sono sottoposti alla legislazione universale; gli atti di straordinaria amministrazione sono definiti dai canoni del Codice in via teorica, mentre in via pratica devono essere individuati in base agli statuti o dai Superiori degli Enti che li pongono in essere.

Occorre infine ricordare che i controlli canonici sugli atti di amministrazione straordinaria, previsti nel Libro V del CIC, riguardano solamente le persone giuridiche pubbliche, poiché solo i beni di una persona pubblica sono da considerarsi beni ecclesiastici ${ }^{16} \mathrm{e}$ quindi devono essere sottoposti al controllo di legittimità dell'Autorità superiore ${ }^{17}$.

Malgrado qualche posizione dottrinale in contrasto con una distinzione così netta, anche durante i lavori della Commissione di studio di revisione del codice del ${ }^{\prime} 17^{18}$, con la formulazione dello schema dell' $80^{19}$ non possono più esserci dubbi: il legislatore accoglie la distinzione (che verrà recepita nel canone 1257) tra "beni ecclesiastici" e "beni non ecclesiastici", appartenenti questi ultimi ad una persona giuridica privata.

Una ulteriore distinzione all'interno degli atti di straordinaria amministrazione si trova poi relativamente a quella speciale categoria di atti che, pur rientrando nell'amministrazione straordinaria e non nell'alienazione, necessitano di un maggior controllo da parte dell'autorità ecclesiastica competente: sono gli atti che vengono definiti come atti assimilati all'alienazione (can. 1295), perché "intaccano il patrimonio della persona giuridica".

\footnotetext{
${ }^{16}$ Cfr. cann. 1256 e 1291.

${ }^{17}$ Porta invece argomenti in contrario, F. AzNAR, La administraciòn de los bienes temporales de la Iglesia, $2^{\circ}$ ed., 1993, pp. 53-54 e pp. 427-431. Secondo questo autore vi sono beni eccelsi astici in senso ampio e beni eccelsi astici in senso stretto: questi ultimi sarebbero quelli di cui sono titolari persone giuridiche pubbliche.

${ }^{18}$ Cfr. A. Mostaza Rodriguez, Derecho patrimonial, in: AA.VV., Derecho canonico, Pamplona 1975, pp. 315-360.

${ }^{19}$ Communicationes 12(1980), pp. 391-392.
} 
Scrive al riguardo J. P. Schouppe che si tratta di valutare "nel concreto" quegli atti che, pur non rientrando nel concetto di alienazione e quindi al controllo dei cann. 1291-1294, siano in concreto in grado di peggiorare il patrimonio della persona giuridica ${ }^{20}$.

\section{Il ruolo degli Organismi di partecipazione verso gli atti} di amministrazione straordinari dell'Ente ecclesiastico: Consiglio diocesano per gli affari economici (CDAE) e Consiglio Parrocchiale affari economici (CPAE)

Veniamo ora all'altro aspetto della nostra ricerca: il ruolo degli organi di partecipazione previsti dal CIC. Come accennato se è rilevante il loro ruolo circa la validità canonica, dell'atto di amministrazione straordinaria o di alienazione posto in essere, lo è a maggior ragione circa l'attuazione del principio di partecipazione dei fedeli al bene della Chiesa e del loro diritto a manifestare le proprie idee ai Pastori (can. $212 \S 3)^{21}$.

Il diritto canonico si preoccupa di assicurare che l'amministrazione dei beni delle persone giuridiche ecclesiastiche non sia rimessa in maniera esclusiva a un singolo e prevede due importanti organismi di collaborazione nella gestione economica del Vescovo, per la diocesi, e di quella della Parrocchia, per il Parroco.

Questi due Organismi di partecipazione in ambito amministrativo, entrambi obbligatori per i rispettivi Enti, sono il Consiglio diocesano per gli affari economici (cann. 492 e 493) e il Consiglio parrocchiale per gli affari economici (can. 537).

In ambito diocesano alcune competenze, circa gli affari economici più rilevanti, sono poi riservate al Collegio dei consultori: per il rilascio di alcune licenze inoltre il Vescovo potrebbe avere l'obbligo di richiedere il parere o il consenso anche di altri enti o persone, in base al diritto universale o proprio o alle tavole di fondazione.

${ }^{20}$ J. P. Schouppe, Elementi di diritto patrimoniale canonico, Milano 1997, pp. 134136. Anche interessante sull'argomento, V. DE PAolis, Negozio giuridico, "quo condicio patrimonialis personae iuridicae peior fieri possit”, Periodica 88(1994), pp. 522-523.

${ }^{21} \mathrm{Su}$ questo argomento, interessante è l'articolo di E. ZANETTI, I fedeli e i beni ecclesiastici: alcune domande, Quaderni di diritto Ecclesiale 4(1991), pp. 305-316. 
Al Consiglio diocesano per gli affari economici e al Consiglio parrocchiale per gli affari economici si aggiunge una norma "generale" (can. 1280) la quale prevede che, anche laddove non siano previsti i due organi suddetti, "ogni persona giuridica abbia almeno due consiglieri che coadiuvano l'amministratore nell'adempimento del suo compito, a norma degli statuti".

Nel primo caso il parere o consenso deve essere richiesto secondo le norme del can. $127 \S 1$, ossia, il Superiore, per porre in essere determinati atti, deve chiedere il consenso o il parere di un collegio o di un gruppo di persone.

Nel secondo caso rientra la norma prevista dal can. $127 \S 2$ : se è richiesto il consenso del gruppo, l'atto è invalido se non lo segue; quando invece si richiede il parere, il Superiore deve solo riceverlo nei modi previsti, ma se ne può anche discostare, purché esista una ragione prevalente che giustifichi questa decisione.

Stabilire quali siano i compiti del Consiglio affari economici diocesano è rimesso al CIC (cann. 493 e 494). Per i Consigli economici delle parrocchie è invece il Vescovo Diocesano a dover emanare il relativo decreto che ne disciplini la struttura e le norme di esercizio della funzione (can. 537).

Dibattuta in dottrina, è la questione se queste disposizioni riguardino solo le persone giuridiche riconosciute, o non anche quelle prive di personalità giuridica autonoma. La soluzione non appare a nostro parere troppo rilevante poiché, comunque, gli atti di alienazione richiedono sempre la licenza canonica del Vescovo diocesano, che sarà coadiuvato dal parere del suo Consiglio affari economici.

Per le persone giuridiche governate da un Collegio la questione non si pone perché sono il Consiglio direttivo o il Consiglio di amministrazione a decidere.

La distinzione tra parere vincolante e non vincolante si attenua in diritto canonico: il can. $127 \S 2,2^{\circ} \mathrm{CIC}$, consiglia infatti all'autorità di non discostarsi dal parere concorde del Consiglio, se non vi sia una ragione di maggior peso, che ne giustifichi il contrario ${ }^{22}$.

${ }^{22}$ Scrive J. I. ARriEtA: „Chi ha chiesto il parere, si è già detto, è tenuto a valutarlo, 
L'importanza del parere consultivo è comprovata dal fatto che in ogni caso il can. 127 determina la nullità dell'atto di governo realizzato senza aver prima ottenuto il parere consultivo richiesto dalle norme.

Nella Diocesi il Codice del 1983 elenca alcuni casi in cui il Vescovo debba preventivamente interpellare i due Consigli, Economico e dei Consultori, in ambito economico, usando il termine "audire debet": la presunzione generale, in Diritto canonico, è infatti che il parere abbia carattere non vincolante, ma in alcuni casi - esplicitamente menzionati dalle norme - il parere è vincolante (de consenso). Questi casi - si noti bene - sono sempre limitati a provvedimenti di carattere economico, per i quali il Vescovo, senza aver interpellato il Collegio dei consultori e il Consiglio affari economici della Diocesi, pone atti invalidi:

- can. 1277: atti di amministrazione straordinaria nei casi della legge universale, nelle tavole di fondazione e nei casi stabiliti dalla Conferenza Episcopale;

- can. $1292 \S 1$ : alienazione di persone giuridiche soggetta all'autorità del Vescovo: il valore è stabilito dal Vescovo con il consenso del CDAE e del Collegio dei consultori. Il Vescovo, inoltre, ha bisogno del consenso dei due organismi collegiali anche per alienare i beni della sua Diocesi.

Sarà la Conferenza Episcopale a stabilire la somma minima per la richiesta della licenza canonica (can. 1292 § 1), nonché il valore della somma massima, oltre la quale viene richiesta la licenza della Santa Sede $^{23}$.

e proprio perciò sembra coerente che - anche se il Diritto non lo impone in forma generale -, l'Autorità che intenda discostarsi dal responso abbia cura di motivare nel testo dell'eventuale decreto (can. $51 \mathrm{CIC}$ ) le ragioni che l'abbiano indotta a seguire una diversa determinazione". Cfr. Discrezionalità e discernimento nel governo della Chiesa, a cura di J. I. Arrieta, Venezia, 2008, p. 146. Per un'analisi della teoria sulla partecipazione, M. Schmitz-W. Schmitt-Glaeser, Art. Partizipation, Staatslexikon IV (1988), pp. 318 ss.

${ }^{23}$ Cfr. per le norme emanate dalle diverse Conferenze episcopali, J.T. MARTIN De Agar, Legislazione delle Conferenze episcopali complementare, Ius Canonicum 32(1992), pp. 173-229. 


\section{Alcune situazioni particolari di atti peggiorativi del patrimonio}

La disciplina degli atti economici richiede dunque una complesso collegamento tra i canoni del codice, la legislazione particolare che li attua e talvolta anche il diritto civile dello Stato. Questa complessità emerge in alcuni casi di atti peggiorativi del patrimonio, che riguardano:

a) Gli Istituti di vita consacrata e le Società di vita apostolica: la Congregazione, in forza del can. $1293 \S 2$, ha introdotto la prassi di richiedere il parere dell'Ordinario del luogo in cui si trova il bene che deve essere alienato. Il can. $635 \S 1$ stabilisce infatti che i beni temporali degli istituti religiosi sono retti dai canoni del Libro V, fatto salvo che non sia espressamente previsto diversamente. In questa ipotesi, tuttavia, la mancata richiesta del parere dell'Ordinario del luogo non ha valore invalidante, ma si configura come un requisito procedurale ${ }^{24}$.

b) La vendita delle reliquie è vietata dal can. $1190 \S 1$, mentre è possibile per le reliquie insigni e le immagini che sono onorate dalla pietà popolare, solo con licenza della Santa Sede. In questo caso ci si trova di fronte a una disciplina non troppo chiara: la regolamentazione non è valida per qualsiasi reliquia o soltanto per quella non autorizzata dalla Santa Sede? La licenza deve essere richiesta solo per l'alienazione o anche per il trasferimento? Come si stabilisce in concreto se una reliquia o un'immagine devono ricadere sotto questa disciplina? ... Questi alcuni dubbi lasciati aperti.

c) Le attività commerciali svolte da un Ente ecclesiastico devono essere sottoposte ai controlli canonici; pertanto la licenza va intesa come integrazione del potere di rappresentanza degli amministratori e come legittimazione di questi ultimi ${ }^{25}$.

\footnotetext{
${ }^{24}$ Congregazione Per GLi ISTITUTI DI VITA CONSACRATA E LE SOCIETA' DI VITA APOSTOLICA, lettera Già da alcuni decenni, rivolta ai Superiori generali sulla documentazione da presentare alla Congregazione nel caso di atti giuridici sui beni temporali, 21 dicembre 2004 (EV 22, anni 2003-2004). Sull'argomento della gestione economica negli Istituti religiosi, E. Arenas-F. Torres, Vita consacrata ed economia, Manuale per l'amministrazione degli Istituti religiosi, Milano, 2006.

${ }^{25}$ Per approfondire la questione, AA.VV., Enti ecclesiastici e controllo dello Stato, a cura di J. I. ArrietA, Venezia, 2007, pp. 206-210.
} 
d) La costituzione di una Fondazione non autonoma. Le Fondazioni pie non autonome sono un insieme di beni che vengono devoluti a una persona giuridica pubblica perché amministri e utilizzi le rendite a fini ecclesiali. Fini fissati dai promotori della Fondazione. Spesso si tratta di far celebrare Sante Messe (can. $1303 \S 1,2^{\circ}$ ). Una persona giuridica pubblica può costituire una Fondazione non autonoma, ma solo previa licenza dell'Ordinario, che il nuovo codice prescrive ad validitatem. L'Ordinario dovrà valutare la capacità di adempiere gli oneri previsti, riguardo al patrimonio disponibile (can. 1304 § 1). Anche su quest'aspetto la normativa universale invita il diritto particolare a dare nuove specificazioni normative (can. $1304 \S 2$ ). Utile ricordare, per inciso, che anche nel caso di una Fondazione privata la vigilanza dell'Ordinario del luogo, perché esecutore di tutte le pie volontà, si estende all'amministrazione (cann. $325 \S 2$ e 1301).

e) Le donazioni e le liberalità. L'Ordinario deve concedere una licenza perché si possano accettare le liberalità gravate da un onere modale o da una condizione (can. 1267 § 2). Rimangono aperti alcuni aspetti relativi alla discrezionalità lasciata all'Ordinario nella decisione nel caso di donazione o testamento modale, che potrebbe confliggere con la volontà dell'Ente destinatario della stessa.

Tenendo in considerazione alcuni recenti casi di discutibile amministrazione delle diocesi, si comprende facilmente la necessità che queste norme non solo siano conosciute, ma anche rese effettivamente operative, a tutela del bene della comunità ecclesiale ${ }^{26}$.

\section{Gli atti di straordinaria amministrazione della Parrocchia:}

\section{la licenza canonica}

Esercitare la responsabilità amministrativa della Parrocchia, sotto l'autorità del Vescovo diocesano, comporta che gli atti amministrativi di maggiore importanza, riguardanti ad esempio la vendita di un bene di valore dell'Ente, richiedano il consenso scritto del Vescovo stesso.

\footnotetext{
${ }^{26}$ Cfr. C. Redaelli, La responsabilità del Vescovo diocesano nei confronti dei beni ecclesiastici, Quaderni, cit., pp. 317-335, e anche J. Herranz CAsado, The personal Power of Governance of the Diocesan Bishop, Communicationes 20(1988), pp. 288-310.
} 
Come sempre, sono due i profili da tenere presenti:

1) Si richiede per tutti gli atti di straordinaria amministrazione la licenza scritta dell'Ordinario (can. $1281 \S 1$ ).

Per la validità delle alienazioni dei beni costituenti il patrimonio stabile (can. 1291) e dei negozi che peggiorino lo stato patrimoniale della persona giuridica (can. 1295), fino al valore stabilito dalla Conferenza Episcopale nazionale, è prevista quindi la licenza del Vescovo, con il consenso del CDAE e del Collegio dei Consultori.

2) Gli atti di straordinaria amministrazione rimandano a:

a) can. 1277 ;

b) altri atti stabiliti dal Vescovo diocesano con un decreto generale, deliberato ai sensi del canone $1281 \S 2$.

Il fatto che il Codice configura il CDAE come un organo consultivo esclude la possibilità di prendere come modello per il suo funzionamento quello solitamente previsto (nell'ordinamento civile e nella prassi comune) per i Consigli di amministrazione dei diversi Enti ${ }^{27}$.

In essi, infatti, pur essendo riconosciuta la funzione di presidenza a una singola persona, tutti gli elementi invece sono quasi completamente posti sullo stesso piano e hanno stessa potestà deliberativa.

Un profilo da approfondire, relativamente alle legislazioni dei singoli Stati, riguarda le conseguenze di diritto civile degli atti di amministrazione straordinaria o di alienazione dei beni del patrimonio dell'Ente ecclesiastico compiuti senza le previste licenze: spesso sono negozi giuridici, non solo canonicamente invalidi o inefficaci, ma che comportano profili di responsabilità civile in capo agli amministratori che li abbiano posti in essere.

\section{Ruolo del Consiglio parrocchiale per gli affari economici (CPAE)}

Il Direttorio Pastorale circa il ministero dei Vescovi, Apostolorum Successores, invita i Vescovi locali a "costituire un Consiglio per gli affari economici, presieduto dal Vescovo o da un suo delegato. Simili

${ }^{27}$ Cfr. A. DE Angelis, I Consigli per gli affari economici: statuti e indicazioni applicative, Monitor Ecclesiasticus 111(1986), p. 60 ss. 
Consigli dovranno costituirsi anche in ciascuna parrocchia e nelle altre persone giuridiche. Per integrare tali organi ci si affiderà a fedeli scelti per la conoscenza della materia economica e del diritto civile, dotati di riconosciuta onestà e di amore per la Chiesa e per l'apostolato..."28.

Si tratta quindi dell'attuazione di quanto già previsto dai canoni del CIC: la costituzione dei Consigli di amministrazione nella diocesi, nelle singole parrocchie e nelle altre istituzioni ed opere diocesane, ammettendovi a partecipare, oltre ai chierici, laici esperti in amministrazione e dotati di onestà e amore per la Chiesa e l'apostolato.

Sarebbe quindi auspicabile che il Vescovo diocesano emani un Regolamento per i Consigli parrocchiali economici (cfr. can. 537 su obbligatorietà del CPAE, che afferma "... in ogni Parrocchia vi sia il Consiglio..."): questo compito si deve far rientrare in quello più ampio del dovere del Vescovo di vigilare sull'amministrazione dei beni delle persone giuridiche sottoposte alla sua giurisdizione (can. 1276) ${ }^{29}$.

La scelta dei componenti che faranno parte del CPAE va compiuta in analogia con quanto stabilito per il CDAE (can. 492 §1): occorrerà però farsi guidare da criteri di buon senso, regolando il numero dei componenti il consiglio in base alla grandezza della comunità parrocchiale, cercando di includervi sempre laici veramente esperti in materie economiche e soprattutto disinteressati ${ }^{30}$.

Il parere del CPAE sugli atti di straordinaria amministrazione dovrà tenere conto non solo dell'opportunità e della correttezza dell'atto in sé ma anche e soprattutto della situazione pastorale ed economica della Parrocchia. Si tratta, comunque, di un parere non vincolante.

${ }^{28}$ Congregazione per i Vescovi, Direttorio per il Ministero Pastorale dei Vescovi, Apostolorum Successores, 22 febbraio 2004, Città del Vaticano 2004, n. 193.

${ }^{29}$ La vigilanza è derivazione del potere di governo, o esecutivo, di cui dispone il Vescovo diocesano; essa si esplica come abbiamo già visto in modo peculiare con la licenza preventiva che gli enti sottoposti al Vescovo devono richiedere per adottare atti di amministrazione straordinaria o di alienazione (can. $1281 \S 1$ ).

${ }^{30}$ Cfr. V. De Paolis, Il Consiglio parrocchiale per gli affari economici e i beni patrimoniali della Parrocchia, in: AA.VV., La Parrocchia, Città del Vaticano 1997, pp. 267-288. 
Scelte diverse, in questo campo, da parte del diritto particolare paiono non auspicabili e, comunque, di scarsa utilità. Il Codice di diritto Canonico prevede infatti già ampie forme di controllo e di tutela sugli atti di straordinaria amministrazione attuati dall'Ente Parrocchia, che devono essere sottoposti alla licenza dell'Ordinario.

La richiesta di un parere vincolante del CPAE avrebbe come unico effetto quello di allungare l'iter burocratico delle pratiche a scapito di una gestione efficiente e agile: sarebbe però opportuno, come suggerisce qualche autore ${ }^{31}$, che il decreto del Vescovo diocesano emanato per regolare i CPAE prevedesse che il Parroco prima di compiere un atto di amministrazione straordinaria, debba richiedere sempre il parere previo.

Sebbene il CPAE svolga una funzione consultiva questo non ne diminuisce l'importanza per la corretta amministrazione della Parrocchia: occorre però sottolineare che per garantire la partecipazione dei fedeli attraverso questo organo, rimane di fondamentale importanza la normativa del Vescovo diocesano che ne regoli compiutamente la composizione e la struttura.

Per le decisioni all'interno del CPAE la ricerca del bene comune deve avvenire non attraverso la "conta" dei voti ma per mezzo di una "comunione di pensiero". Questo quindi esprime una natura ecclesiale di grande valore del CPAE: pur rimanendo sempre il Parroco, unico amministratore e responsabile della gestione ${ }^{32}$, il Consiglio affari economici della Parrocchia diviene uno dei luoghi dove i fedeli possono manifestare la loro collaborazione per il bene della comunità ecclesiale.

\section{Conclusioni}

Nella materia della gestione patrimoniale amministrativa è necessario ricercare un delicato, e spesso complesso, equilibrio tra la preoccupazione di tutelare il patrimonio ecclesiastico e la necessità di garantire una buona gestione dello stesso.

${ }^{31}$ Così G. Morgante, L'amministrazione dei beni temporali della Chiesa. Diritto canonico. Diritto concordatario. Pastorale, Casale Monferrato 1993, p. 121.

${ }^{32}$ F. Coccopalmerio, De paroecia, Roma 1991, p. 205. 
La grande novità introdotta dal più rilevante tra gli atti peggiorativi del patrimonio, ossia l'alienazione, è l'essere passati dal concetto di inalienabilità dei beni ecclesiastici (che venivano così in qualche modo considerati quasi "sacri") al concetto di buona gestione del patrimonio. L'alienabilità deve così essere talvolta prevista al fine di garantire una buona gestione amministrativa ed economica dei beni ecclesiastici.

L'ordinamento canonico ha così cercato di realizzare questi due obiettivi, talvolta in conflitto, prevedendo un sistema di controlli e di condizioni da parte delle Autorità superiori.

Come abbiamo cercato di mettere in rilievo, con il nuovo CIC, un ruolo sempre più importante assumono i Consigli posti a sostegno degli amministratori dei beni, primo tra tutti il Vescovo diocesano, cui il Codice dell' 83 per gli atti più rilevanti affianca due Consigli: quello degli Affari economici e quello dei Consultori. Non tenere conto di queste norme ha generato talvolta seri problemi ai patrimoni ecclesiali: la preziosa funzione di questi organi che coadiuvano il Vescovo può venire vanificata se non si osserva quanto prescrive il can. 1282: agli amministratori dei beni ecclesiastici è fatto obbligo di adempiere i loro compiti in nome della Chiesa e secondo i principi e le norme del Diritto Canonico.

E' stato, altresì, introdotto, con l'espressione "alienazione e atti assimilati", un controllo su quegli atti che pur non rientrando nel concetto restrittivo del can. 1291 ("alienazioni di beni del patrimonio stabile") comportano, comunque, un pregiudizio alla situazione patrimoniale della persona giuridica (can. 1295).

Anche questa evoluzione non è di poca importanza per l'amministrazione del patrimonio degli Enti ecclesiastici, soprattutto perché mostra un passo in avanti fondamentale: il voler passare da un concetto statico, a nostro parere non più sufficiente, di compilare il rendiconto amministrativo a fine esercizio, ad un concetto dinamico e moderno di gestione del patrimonio.

Fatto più che mai indispensabile per un'attenta gestione delle risorse degli Enti ecclesiastici, a cominciare dalle Diocesi, dalle Parrocchie, per finire ai patrimoni (talvolta molto ingenti) gestiti dagli Istituti 
religiosi ${ }^{33}$. Un progresso, questo, al cui servizio sono posti i Consigli economici, ma che deve tradursi in pratica quotidiana.

Un Ente ecclesiastico non può realizzare atti peggiorativi del patrimonio ecclesiastico, o limitandolo o mettendolo in pericolo, senza che la comunità ecclesiale ne sia in qualche modo consapevole e partecipe. Atti che possono andare dalla costituzione di un diritto reale sul bene immobile all'assunzione di personale: si tratta di atti che ora il Codice Canonico non considera residuali, definendoli al can. 1277 quali “... atti di maggiore importanza, e come tali sottoposti a particolari controlli".

Alla luce della legislazione del Codice, ai documenti del Concilio Vaticano II che il CIC/83 trasferisce in norme giuridiche, nonché agli orientamenti successivi delle Congregazioni e delle singole Conferenze Episcopali ${ }^{34}$, si deve riconoscere alla funzione amministrativa, con cui l'autorità gestisce i beni ecclesiali, una sicura valenza ecclesiologica.

Si tratta di un compito di governo che ha una indubbia rilevanza pastorale: come tale non può essere affrontato con superficialità o quale necessità contingente, ma merita una considerazione e un'attenzione del tutto particolare.

Ma non solo. Trattandosi di un'attività di rilevanza ecclesiale, nel campo economico dovrà essere realizzata sempre più la partecipazione e la collaborazione dei fedeli laici ${ }^{35}$ : come abbiamo cercato di esami-

${ }^{33}$ M. Grumo, Gestione, bilancio e sostenibilità economica degli enti ecclesiastici, Milano 2010.

${ }^{34} \mathrm{Si}$ pensi ad esempio alle due Istruzioni in materia amministrativa, del 1992 e poi del 2005, emanate dalla Conferenza Episcopale Italiana. Cfr. ConfErenza Episcopale ITALIANA, Istruzione in materia amministrativa, 1 aprile 1992, Notiziario CEI 3(1992), pp. 60-143 e 1 settembre 2005, Notiziario CEI 8/9(2005), pp. 329-422.

${ }^{35} \mathrm{La}$ partecipazione è lo strumento che attua e realizza la comunione ecclesiale, segno e testimonianza fondante di una comunità di fedeli perché, come si legge nella Costituzione Lumen Gentium del Concilio Vaticano II, lo Spirito „dispensa pure tra i fedeli di ogni ordine grazie speciali, con le quali li rende adatti e pronti ad assumersi vari incarichi e uffici utili al rinnovamento e alla maggiore espansione della Chiesa". Costituzione Dogmatica sulla Chiesa, Lumen Gentium, 21 novembre 1964, AAS 57(1965), pp. 5-71, al nr. 12. 
nare il Codice già lo prevede, mediante Organi e strutture ben definite che se applicate correttamente potranno garantire una sempre più competente e corretta amministrazione dei beni della Chiesa, sia evitando gli scandali provocati per la cattiva gestione economica a cui abbiamo purtroppo assistito più volte recentemente, sia realizzando una non marginale testimonianza dei principi evangelici.

\section{The role of organizations in the administration in the Code of Canon Law: the acts of extraordinary administration and alienation of ecclesiastical organizations}

The administration of the goods of ecclesiastical organizations is a complex and very delicate task. The practise often shows us how rules of the Code of Canon Law is still partly unclear in order to have a truly effective execution for the protection of institutions assets.

In 1983 the CIC on one hand seeks to protect the assets of the institution, giving rules in order to make sure the institution assets: the distinction between acts of extraordinary administration and acts of alienation tries to prevent the assets from suffering an unmotivated decrease.

On the other hand, the new code introduces a number of organizations cooperating in the administration, not to leave the administrator without a helping hand. These organisms are first of all to protect those who are responsible for the administration of goods, not only to control their work. This control is accomplished primarily through the authorization canonical acts, intended for all those acts which go beyond the ordinary.

However, these aspects still need more completeness in regulation on one hand; on the other hand they need a more effective execution in practice: these two aspects are closely linked. 